\title{
Design of an emittance exchanger for production of special shapes of the electron beam current
}

\author{
Dmitry Yu. Shchegolkov and Evgenya I. Simakov \\ Los Alamos National Laboratory, P.O. Box 1663, Los Alamos, New Mexico 87545, USA
}

(Received 5 November 2013; published 28 April 2014)

\begin{abstract}
Recently, considerable attention has been focused on electron beam current profile shaping by means of a transverse beam mask followed by an emittance exchanger (EEX). This setup can transform a transverse particle distribution into a longitudinal particle distribution. We investigate the EEX technique with application to production of a double triangular drive and a trapezoidal main bunches for high transformer ratio, high brightness dielectric wakefield accelerators. We perform numerical optimization of two realistic configurations: a double dogleg and a chicane. In this paper we report sample designs and discuss the effects of the beam line nonlinearities, beam space charge, and coherent synchrotron radiation on a current profile of the output beam.
\end{abstract}

DOI: 10.1103/PhysRevSTAB.17.041301

PACS numbers: 29.20.Ej, 41.75.Lx, 41.75.Ht, 41.85.Ct

\section{INTRODUCTION}

Many free electron laser (FEL) sources of infrared to $\mathrm{X}$-ray coherent radiation have been built or are planned to be built across the world [1-6]. The essential constitutive part of each FEL is an electron accelerator. The lasing efficiency in the undulator and the shortest achievable wavelength are determined by the quality of the electron bunch produced by the accelerator: bunch current, width, particle energy, and energy spread. Thus almost any FEL performance or frequency range enhancement inevitably sets new challenges to the accelerator technology [7]. These challenges and the desire to minimize the size of FELs have triggered research into alternative accelerator schemes like dielectric wakefield acceleration [8]. As opposed to the conventional superconducting rf technology, the potential of dielectric wakefield accelerators has never been fully exploited.

An x-ray FEL is going to be a part of the Los Alamos National Laboratory (LANL) future signature facility Matter-Radiation Interactions in Extremes (MaRIE) [9-11]. The preconceptual design for MaRIE is underway at LANL, with the design of the electron linear accelerator being one of the main research tasks. The existing space constraints of the mountainous terrain dictate that the final energy of the electron beam for the $\mathrm{x}$-ray free-electron laser (XFEL) using available $S$-band room temperature technology is no higher than $12 \mathrm{GeV}$. The number and the energy of photons produced by the XFEL, however, is strongly dependent on the electron energy, with the more energetic beam delivering more energetic photons to the user. Although generally the baseline design needs to be conservative and

Published by the American Physical Society under the terms of the Creative Commons Attribution 3.0 License. Further distribution of this work must maintain attribution to the author(s) and the published article's title, journal citation, and DOI. rely on existing technology, any future upgrade would immediately call for looking into advanced accelerator concepts capable of boosting the electron beam energy up by a few $\mathrm{GeV}$ in a very short distance without degrading the beam quality. Dielectric wakefield accelerators (DWAs) $[12,13,8]$ have the potential to satisfy the requirements set for the MaRIE linac afterburner, which are the acceleration gradient of above $100 \mathrm{MV} / \mathrm{m}$ and the gained beam energy spread of less than $0.1 \%$ [6]. DWAs are formed by one or several coaxial dielectric layers surrounded by a metal cladding [12]. Wakefields in dielectric structures may reach gradients on the order of $10 \mathrm{GV} / \mathrm{m}$ [14] with $100 \mathrm{MV} / \mathrm{m}$ being demonstrated in multiple experiments $[15,16]$. They also have the remarkable property that the wakefield's axial electric field and the transverse electric field are transversely uniform and linear, respectively. This is due to the fact that the relativistic drive beam and the subsequent wakefield travel very nearly at the speed of light. If one can make the wakefield to be longitudinally constant along the bunch it will result in no induced energy spread within the bunch, leading to an extraordinary condition of preserving the main beam brightness while providing high gradient acceleration. We have realized that it is possible for an accelerated beam in a DWA to achieve the needed small energy spread if the longitudinal witness bunch profile is properly customized [17].

An important characteristic of the wakefield acceleration is the transformer ratio (TR). It is the ratio of the peak accelerating gradient to the peak decelerating gradient experienced by the drive bunch, and thus it determines how long the drive bunch will be stable before some part of it decelerates to low energies. For a finite length longitudinally symmetric bunch the TR can never exceed 2 $[18,19]$. An enhanced TR can be achieved with a ramped beam or a ramp-profiled bunch train [18,20]. A high transformer ratio has been experimentally demonstrated with a bunch train [21]. Recently, a double triangular (DT) 


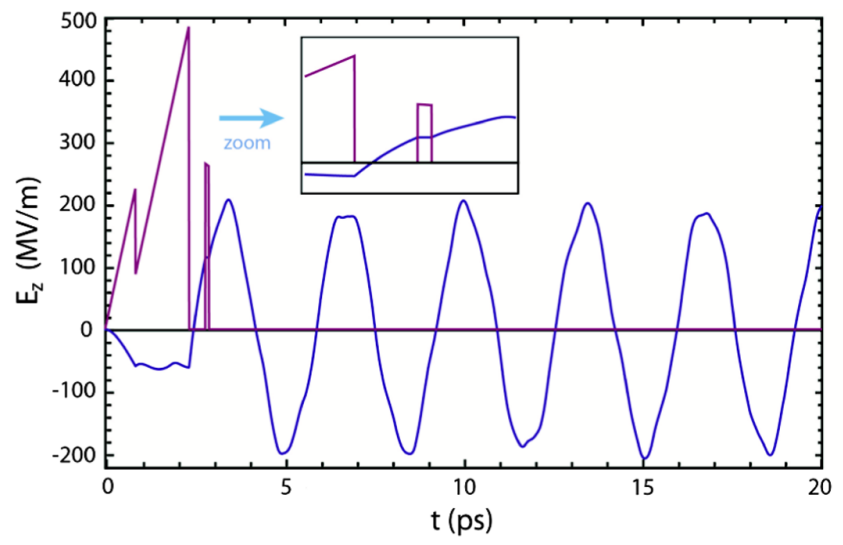

FIG. 1. The wakefield (blue) excited by the optimized current profile (red) with an enhanced transformer ratio. Inset: the wakefield inside of a trapezoidal witness bunch (zoomed in), showing a flat gradient observed by the witness bunch.

beam shape was proposed, making it possible to achieve high TRs with almost uniform drive bunch deceleration [22]. Both LANL and Argonne National Laboratory have independently proposed to experimentally demonstrate for the first time generation of a DT drive beam and a high transformer ratio [23].

The current profile in a DT bunch (shown in Fig. 1) is described by the following formula:

$$
I(t)= \begin{cases}I_{0} \omega t, & 0<t<\frac{\pi}{2 \omega} \\ I_{0} \omega t-I_{0}, & \frac{\pi}{2 \omega}<t<T .\end{cases}
$$

Here $\omega$ is the frequency of the wake mode to be excited, $T$ is the bunch duration and $I_{0}$ is the charge normalizing coefficient. The maximum achievable TR is then

$$
\mathrm{TR}=\sqrt{1+(\omega T-1)^{2}} .
$$

The TR is approximately proportional to the beam length in wavelengths of the induced wakefield radiation and thus can be made very large.

It has to be realized that DT bunch shapes are difficult to produce. The standard techniques to modulate the beam current by means of electric or magnetic field changes are too slow for shaping pulses on the millimeter scale necessary for a DWA operating at $100 \mathrm{GHz}$ and above. Modulating a photocathode current by means of laser beam modulation was proven not to produce sharp longitudinal beam profiles either, due to the difference in the space charge effects strongly affecting beam propagation at low energies [24]. A fast technique is used at the Accelerator Test Facility at Brookhaven National Laboratory [25,26]. It utilizes an energy chirped beam which is cut with a transverse beam mask placed inside of the beam line dispersive region where one transverse coordinate is linearly related to both the particle energy and arrival time.
The drawback of this technique is that the shaped beam is also chirped. The focus of this paper is the relatively new technique based on an emittance exchange between the transverse and longitudinal phase spaces, which does not need the beam to be energy modulated and thus can be used for high brightness beams [27-31]. The EEX optics were initially proposed in application to the free electron laser for transverse emittance reduction in an electron beam with a smaller longitudinal emittance [29] and first verified experimentally at the Fermilab A0 Photoinjector [32]. In general, the EEX is a device which swaps longitudinal beam emittance with one of the transverse emittances, leaving the third emittance unchanged. However by using enough quadrupole magnets before and (or) after the EEX one can realize more specific transformations, one of which is converting a transverse particle distribution into a longitudinal one. There are a number of papers written about different EEX designs [28,30,31]; however, they do not address nonlinear effects in the EEX and the specifics of achieving the narrow energy spectrum required for FELs. Those are the effects that we focus on in this paper.

We present the results of the EEX optimization accounting for the beam line nonlinearities and demonstrate in simulations the production of a double triangular drive beam and a trapezoidal witness bunch out of a single Gaussian beam.

\section{REDUCING ENERGY SPREAD BY CURRENT SHAPING}

Bunch shaping can address the problem of induced energy spread in the accelerated, also called witness, beam. Similar to the drive bunch shaping, some optimization of the witness beam can be used to satisfy the additional requirements on uniformity of the accelerating gradient within the witness bunch. We have shown that proper timing between the DT drive beam and the witness bunch together with a custom trapezoidal shape of the witness bunch can be used to reduce the witness beam energy spread, and, in doing so, preserve the beam brightness [17]. Figure 1 shows the proposed time profiles of the drive and accelerated bunches for a possible proof-of-principal experiment.

The wakefield was computed using the Green's function method for the electron bunch moving at the speed of light [13], which is a good approximation when electron energy is a few $\mathrm{MeV}$ or higher. The computed wakefield is the sum of the five lowest propagating waveguide eigenmodes with excitation coefficients. The tube dielectric material was assumed to be silica with the outside metal cladding and tube parameters from Table I resulting in a cutoff frequency of about $300 \mathrm{GHz}$. The wakefield of Fig. 1 is generated by an on-axis electron beam with the beam current parameters given in Table I.

The computation indicates that a drive charge of $5 \mathrm{nC}$ can produce gradients as high as $200 \mathrm{MV} / \mathrm{m}$. For a 
TABLE I. The parameters for the dielectric tube and electron bunches for a DWA at $300 \mathrm{GHz}$.

\begin{tabular}{lc}
\hline \hline Dielectric permittivity & 3.75 \\
Tube inner diameter & $1.14 \mathrm{~mm}$ \\
Tube outer diameter & $1.324 \mathrm{~mm}$ \\
Trapezoidal bunch length & $t_{0}=0.1 \mathrm{ps}$ \\
Trapezoidal bunch current & $A \cdot\left(t_{s}-t\right), t_{1} \leq t \leq t_{1}+t_{0}$, \\
& $t_{1}=2.8017 \mathrm{ps}, t_{\mathrm{s}}=t_{1}+6 \mathrm{ps}$ \\
Trapezoidal bunch charge & $0.25 \mathrm{nC}$ \\
DT bunch length & $T=2.35 \mathrm{ps}$ \\
DT bunch charge & $5 \mathrm{nC}$ \\
DT bunch start time & $0 \mathrm{~ns}$ \\
First wake-mode frequency & $\omega=1872 \mathrm{~ns}^{-1}(298 \mathrm{GHz})$ \\
\hline \hline
\end{tabular}

conventional metal-accelerating structure gradients of this value would be well above the breakdown limit. In this example the calculated energy spread in the witness bunch is below $10^{-5}$ (Fig. 1). Note that even though a relatively short drive bunch is used for the sake of simplifying the future experiment, the TR is still about 4 . However, the accelerating gradient is not fully utilized in this configuration, since in order to satisfy the low-energy spread requirement, the witness bunch had to be moved to the area with a positive gradient slope.

\section{BEAM SHAPING WITH AN EEX}

To produce custom beam currents it was proposed to use an emittance exchanger which translates the transverse particle distribution (along the $\mathrm{x}$-coordinate) into the same longitudinal (z-coordinate) distribution, with some scaling factor. For the simple proof-of-principal experiment we would like to be able to produce both the drive bunch and the witness bunch from the same initial beam, though it may not be the case for a real accelerator afterburner. First, the beam particle distribution along one of the transverse coordinates has to be shaped to match the required longitudinal beam (current) profile with some scaling factor. We assumed the initial beam to be Gaussian in all coordinates of the 6-dimensional phase space with the parameters shown in Table II, which are close to those

TABLE II. Parameters of the Gaussian electron beam used in Elegant simulations.

\begin{tabular}{lc}
\hline \hline Parameter & Value \\
\hline$\gamma \cdot \beta$ & $78.28(E=40 \mathrm{MeV})$ \\
$\varepsilon_{\mathrm{nx}}$ & $5.33 \times 10^{-6} \mathrm{~m}$ \\
$\varepsilon_{\mathrm{ny}}$ & $10 \times 10^{-6} \mathrm{~m}$ \\
$\sigma_{\mathrm{dp}}$ & 0.001 \\
$\sigma_{\mathrm{s}}$ & $0.0012 \mathrm{~m}$ \\
$\alpha_{\mathrm{x}}$ & 0 \\
$\alpha_{\mathrm{y}}$ & 0 \\
$\beta_{\mathrm{x}}$ & $3 \mathrm{~m}$ \\
$\beta_{\mathrm{y}}$ & $3 \mathrm{~m}$ \\
\hline \hline
\end{tabular}

predicted for the new Fermilab ASTA facility [33]. We will not discuss here what the reasonable size and thickness of the mask have to be to provide the high contrast of the beam profile. We assume that the mask is ideal, i.e., particles hitting the mask are fully intercepted by the mask and the rest of them freely propagate through the opening. In reality the particles which hit the mask may be just scattered and only later intercepted by the walls of the beam line. In order to obtain the desired beam profile shown in Fig. 1 we derived the shape for the mask optimized for retaining the maximum number of particles in the assumption that the initial beam had Gaussian distribution along both transverse coordinates with known beam widths. In our case $55 \%$ of particles are intercepted by the mask and lost. A simulation with a finite number of particles inevitably introduces some small statistical deviations and discretization effects. Figure 2 shows the mask placed on top of a Gaussian distribution of particles, a simulated transverse particle distribution directly after the mask and a corresponding one-dimensional particle density along $\mathrm{x}$. We used Elegant [34] to simulate the initial particle distribution with the parameters listed in Table II. A script was written for Elegant to simulate the mask.

Without taking into account the space charge effects and coherent synchrotron radiation (CSR), for each particle the final 6-dimensional phase space coordinates in Elegant can be obtained from the initial phase space coordinates while accounting for up to the third-order effects using a transfer matrix of the beam line:

$$
\begin{aligned}
& X_{i}^{F}=R_{i, j} X_{j}+T_{i, j, k} X_{j} X_{k}+Q_{i, j, k, l} X_{j} X_{k} X_{l} ; \\
& i, j, k, l=1,2, \ldots 6 .
\end{aligned}
$$

To convert $x-y$ into $z-y$ distribution in the first (linear) order (described by $R$ matrix), we want to have all $R_{5, j}=0$ except for $R_{5,1}$, which determines the stretch factor.

One of the easiest schemes that can do this transformation is shown in Fig. 3 and consists of a set of quadrupole magnets, two identical doglegs, a deflecting cavity, and a fundamental mode cavity in between the doglegs. The conditions $R_{5,3}=R_{5,4}=0$ are satisfied automatically as the y space remains uncoupled to both $\mathrm{x}$ and $\mathrm{z}$ spaces. In order to make $R_{5,5}=R_{5,6}=0$ the dispersion of the doglegs has to be matched to the dispersion of the deflecting cavity. Accounting for the finite length of the deflecting cavity makes it necessary to include a fundamental mode cavity to fully satisfy this condition. The fundamental mode cavity compensates for a dispersive energy gain in the deflecting cavity [30].

Matrix elements $R_{5,1}$ and $R_{5,2}$ can be adjusted using quadrupole magnets. Indeed, using just two quadrupoles is enough to set $R_{5,2}=0$ and $R_{5,1}$ to any arbitrary value within some limits. However with only two quadrupoles we do not get control over the beam width, and since the higher order terms in the transfer matrix may become large, they 


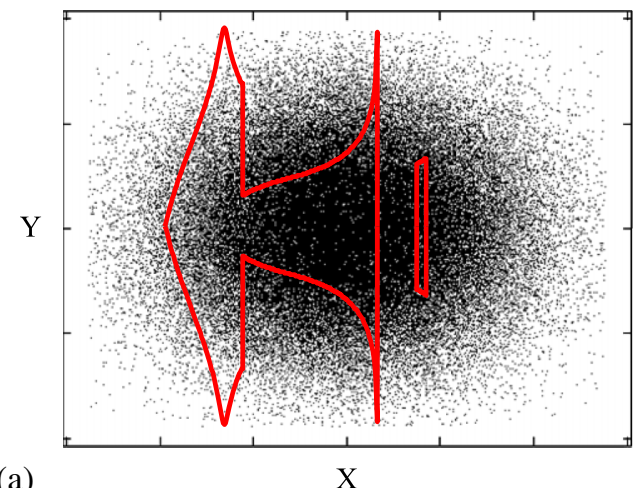

(a)

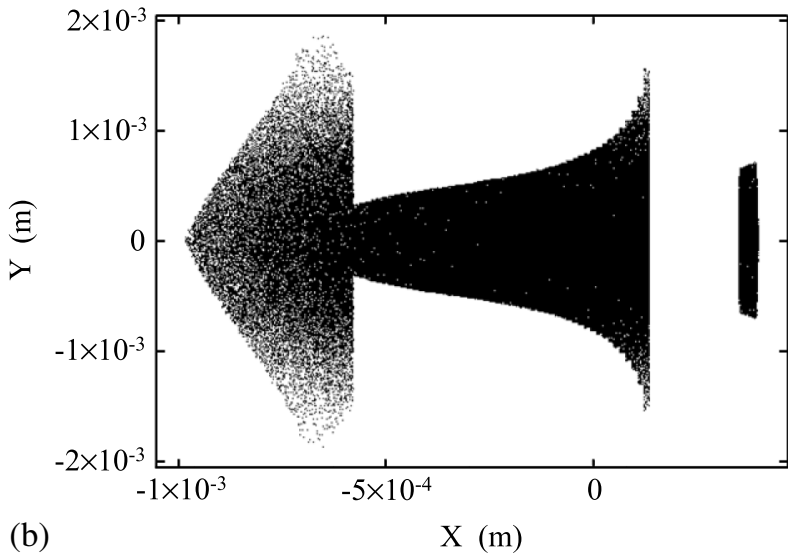

Linear density of particles (arb. units)

(b)

$\mathrm{X}(\mathrm{m})$

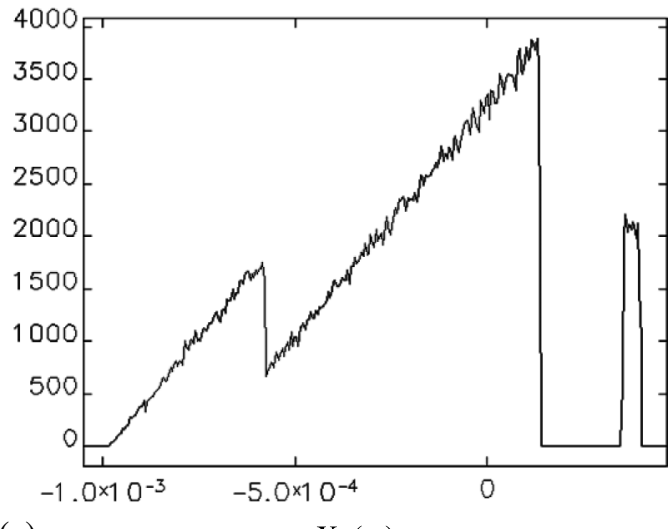

(c)

$\mathrm{X}(\mathrm{m})$

FIG. 2. Illustration of shaping the beam profile with a beam mask at the EEX input to subsequently produce both the drive and the main bunches. The shape of the mask and its orientation with respect to the beam for producing a DT and a trapezoidal $x$ distribution from a Gaussian beam (a). Distribution of particles simulated in Elegant [35] after passing the initial Gaussian beam through the mask (b). Corresponding integral particle distribution along $x$ (c).

may severely alter the output current pulse in this case as shown in Fig. 4(a). The other drawback of using only two quadrupoles is having no control over the $R_{6,1}$ matrix element which in the EEX beam line can produce a big energy chirp. As an example, in Fig. 4(b) the total induced energy spread is about $3 \%$ and the energy spread in the witness bunch itself is $0.11 \%$ that is above the acceptable value for a proposed MaRIE linac afterburner.

We use four quadrupole magnets in the beam line to have more flexibility in magnet currents to minimize the

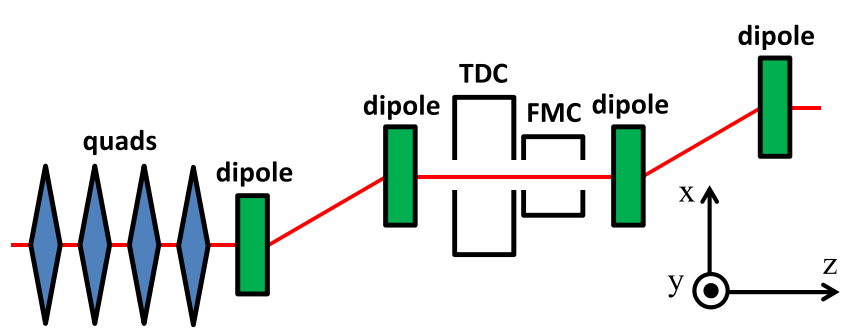

FIG. 3. Emittance exchanger in a double dogleg configuration. The abbreviations TDC and FMC denote "transverse deflecting cavity" and "fundamental mode cavity." unwanted effects. First of all, we minimize transfer matrix terms responsible for nonlinear effects. Although the transfer matrix does not depend on the initial beam parameters, a bigger beam is more prone to nonlinearities of the beam line elements. Thus we impose the condition of having peak values of beam widths inside of the EEX as small as possible. Second, to keep the beam energy spread low we also limit the $\left|R_{6,1}\right|$ to restrict the longitudinal momentum chirp to the acceptable limits. That being said, even four quadrupoles is not enough to satisfy all the requirements, so we relax the value of $R_{5,1}$, as it is always possible to adjust the beam size at the mask and the mask itself to produce a current pulse of a proper length at the EEX output.

Although the double dogleg EEX configuration is the easiest one, for many accelerator beam facilities it is considered not acceptable. For example, if the EEX is to be integrated into the existing beam line it is desirable that it does not shift the downstream end of the beam line. This is where using a more sophisticated chicane configuration becomes necessary $[29,30]$. In a chicane, the output beam is aligned with the initial beam. The simplest version of the chicane EEX, suggested in [29], can provide only partial emittance exchange because of nonzero cross-plane matrix 
Particle flux (arb. units)

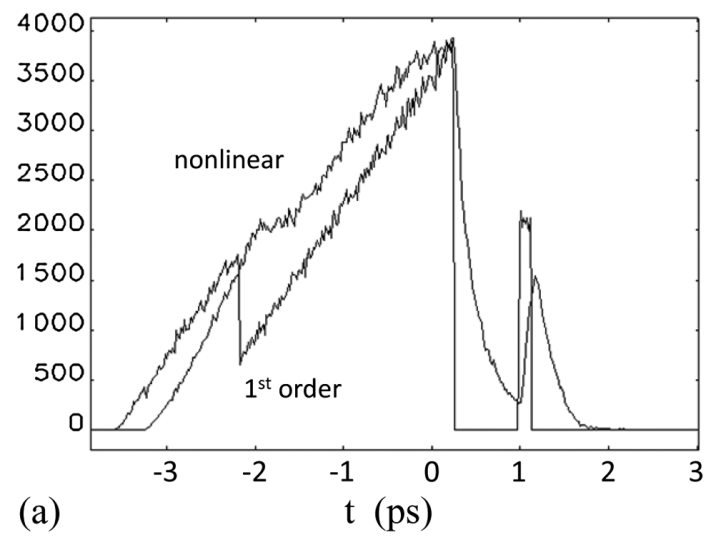

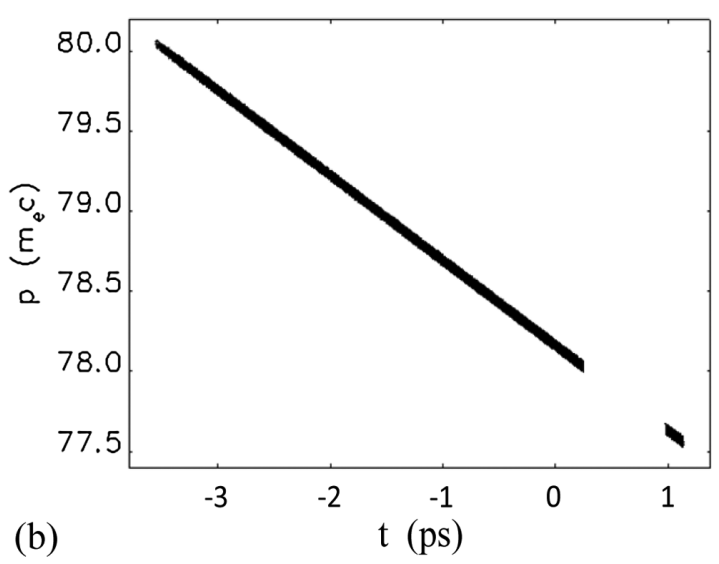

FIG. 4. The beam profile at the output of a double dogleg EEX preceded by two quadrupoles with and without accounting for the nonlinear order transfer matrix terms. The stretch (compression) factor, $R_{5,1}$, is set to $R_{5,1}=1$. Although the output beam profile looks ideal in the first order, in reality there are strong distortions due to nonlinearities in the beam line (a). The corresponding momentum chirp of the output beam computed in first order, $R_{6,1}=-23 \mathrm{~m}^{-1}$ (b).

elements in the linear order. However it was pointed out in [30] that chicane configurations can also provide perfect emittance exchange if two additional quadrupole magnets are used in between two doglegs, as shown in Fig. 5. Using the chicane configuration is also preferable as it allows switching the EEX on and off without changing the output beam trajectory, thus allowing more direct observation of the EEX effect. Our experience of simulating both chicane and double dogleg configurations shows that both schemes are very similar in performance with respect to the emittance exchange, and both of them suffer from similar nonlinearities. Further on in this paper we will focus on the chicane configuration.

\section{NUMERICAL OPTIMIZATION OF THE EEX}

Following [30], to get the perfect emittance exchange to first order we insert two identical quadrupole magnets with a spacing of twice their focusing distance after the first dogleg (Fig. 5). This way the dispersion produced by the first dogleg changes sign as if the dogleg is flipped.
Again, we used initial beam parameters from Table II. During optimization we considered all beam line spacings between the elements fixed and only optimized magnets' currents and cavities' voltages. The optimized parameters of the EEX in the chicane configuration are shown in Table III. The abbreviations used in the table are as follows: K1: geometric quadrupole strength; HGAP: half-gap between poles; FINT: edgefield integral; E1: entrance edge angle; and E2: exit edge angle. The stretch factor came out to be close to 1 , $R_{5,1}=1.06$.

Even though to first order the system works perfectly, accounting for higher order effects distorts the current profile. Figures 6(a)-(c) show the beam after passing through the EEX. The output transverse profile is Gaussian [Fig. 6(a)]. The total energy spread is $0.09 \%$ [Fig. 6(b)], and the chirp coefficient $R_{6,1} / R_{5,1}=$ $-0.072 \mathrm{~m}^{-1}$. The output current profile looks close to the desired one, but it has smoothed edges, and the main beam trapezoid has a slope in the wrong direction [Fig. 6(c)]. The analysis of the second-order elements, described by T-matrix, reveals that the observed aberrations

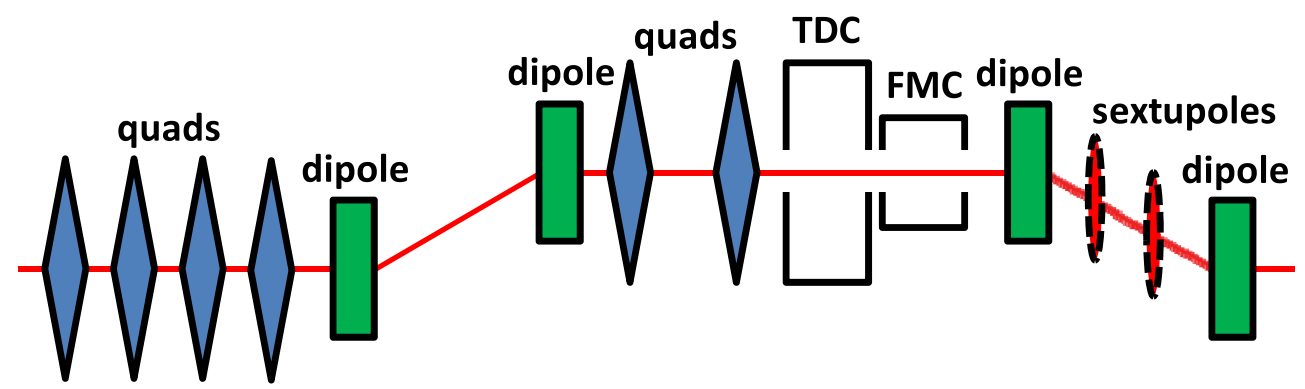

FIG. 5. Emittance exchanger in the chicane configuration. The abbreviations TDC and FMC stand for "transverse deflecting cavity" and "fundamental mode cavity." 
TABLE III. Optimized parameters of the chicane type EEX.

\begin{tabular}{|c|c|c|c|}
\hline Element sequence & Element name & Length (m) & Values of parameters \\
\hline 1 & Mask & 0 & \\
\hline 2 & Drift space & 0.1 & \\
\hline 3 & Quadrupole magnet & 0.167 & $K 1=10.5 \mathrm{~m}^{-2}$ \\
\hline 4 & Drift space & 0.2 & \\
\hline 5 & Quadrupole magnet & 0.167 & $K 1=24.6 \mathrm{~m}^{-2}$ \\
\hline 6 & Drift space & 0.2 & \\
\hline 7 & Quadrupole magnet & 0.167 & $K 1=-22.3 \mathrm{~m}^{-2}$ \\
\hline 8 & Drift space & 0.2 & \\
\hline 9 & Quadrupole magnet & 0.167 & $K 1=17.9 \mathrm{~m}^{-2}$ \\
\hline 10 & Drift space & 0.1 & \\
\hline 11 & Dipole magnet & 0.305 & $E 2=18 \mathrm{deg} ; \mathrm{HGAP}=0.029 \mathrm{~m} ; \mathrm{FINT}=0.59$ \\
\hline 12 & Drift space & 1.172 & \\
\hline 13 & Dipole magnet & 0.305 & $E 1=-18 \mathrm{deg} ; \mathrm{HGAP}=0.029 \mathrm{~m} ; \mathrm{FINT}=0.59$ \\
\hline 14 & Drift space & 0.1 & \\
\hline 15 & Quadrupole magnet & 0.167 & $K 1=10.8 \mathrm{~m}^{-2}$ \\
\hline 16 & Drift space & 1.0 & \\
\hline 17 & Quadrupole magnet & 0.167 & $K 1=10.8 \mathrm{~m}^{-2}$ \\
\hline 18 & Drift space & 0.1 & \\
\hline 19 & Deflecting cavity & 0.1923 & Freq $=3.9 \mathrm{GHz} ; U=978762 \mathrm{~V} ;$ Phase $=-90$ \\
\hline 20 & FM cavity & 0.115 & Freq $=1.3 \mathrm{GHz} ; U=188131 \mathrm{~V} ;$ Phase $=0$ \\
\hline 21 & Drift space & 0.1 & \\
\hline 22 & Dipole magnet & 0.305 & $E 2=-18 \mathrm{deg} ; \mathrm{HGAP}=0.029 \mathrm{~m} ;$ FINT $=0.59$ \\
\hline 23 & Drift space & 1.172 & \\
\hline 24 & Dipole magnet & 0.305 & $E 1=18 \mathrm{deg} ; \mathrm{HGAP}=0.029 \mathrm{~m} ; \mathrm{FINT}=0.59$. \\
\hline
\end{tabular}

are due to the second-order matrix elements $T_{5,5,5}=$ $5.1 \mathrm{~m}^{-1}$ and $T_{5,4,4}=71 \mathrm{~m}$.

The two $T$-matrix elements causing main aberrations can be eliminated by inserting two sextupole magnets as shown in Fig. 5 with dashed contours. However the required sextupole currents are quite high, and in turn may cause some other nonlinearities to grow. For two sextupole magnets of $L=0.167 \mathrm{~m}$ length equally separated from each other and the dipoles, the geometric strengths which make $T_{5,5,5}$ and $T_{5,4,4}$ negligibly small are $K_{2}=167 \mathrm{~m}^{-3}$ for the upstream sextupole and $K_{2}=$ $-188 \mathrm{~m}^{-3}$ for the downstream sextupole. Notably, these sextupoles do not give any essential rise to other $T_{5, \mathrm{i}, \mathrm{j}}$ elements. The output beam current in the system with sextupoles is shown in Fig. 6(d), the current profile now demonstrates sharper back edges and a flatter top of the witness bunch instead of a pronounced slope in a wrong direction.

To understand where the rest of the aberrations come from, and whether they come from third-order effects, we computed the shapes of the output current pulse using a full beam line transfer matrix calculated by Elegant and restricting the elements up to second and up to third order, respectively. The corresponding current profiles are shown in Fig. 7. In the second-order case [Fig. 7(a)] the current pulse shape looks very similar to the desired current profile that fully agrees with $T_{5, \mathrm{i}, \mathrm{j}}$ elements being negligibly small. Accounting for the third-order elements does not add noticeable aberrations but only results in slightly smoothened edges [Fig. 7(b)]. This leads us to the conclusion that aberrations of Fig. 6(d) are not due to any of the second- or third-order matrix elements. The explanation of this is that although Elegant is a matrix code it uses a kicks method when simulating particle propagation through a deflecting cavity. However, for calculating the full beam line matrix Elegant reduces the radio-frequency deflecting cavity element to a first-order matrix and does not compute second- and third-order elements for the deflecting cavity. To further optimize the beam line, a full matrix representation of the radiofrequency deflecting cavity element is required.

The higher order elements in the matrix of a deflecting cavity strongly depend on the particular cavity design. Instead of implementing a higher order expansion matrix for an idealized cavity model with linearly dependent fields on the transverse coordinates utilized in Elegant, we collaborated with a team from Tech-X Corporation [36] to derive second- and third-order matrix elements for a real LANL's $\mathrm{TM}_{110} 15 \mathrm{~cm}$ long $1.3 \mathrm{GHz}$ standing wave cavity with a close deflecting strength using nonidealized, numerically simulated fields inside of the cavity. The fundamental cavity voltage and the optimum magnets' currents of the entire EEX system had to be retuned to accommodate for a different cavity. The same approach allowed us to satisfy the requirements set for $R$-matrix elements and to minimize second-order nonlinearities with a pair of sextupoles inside 

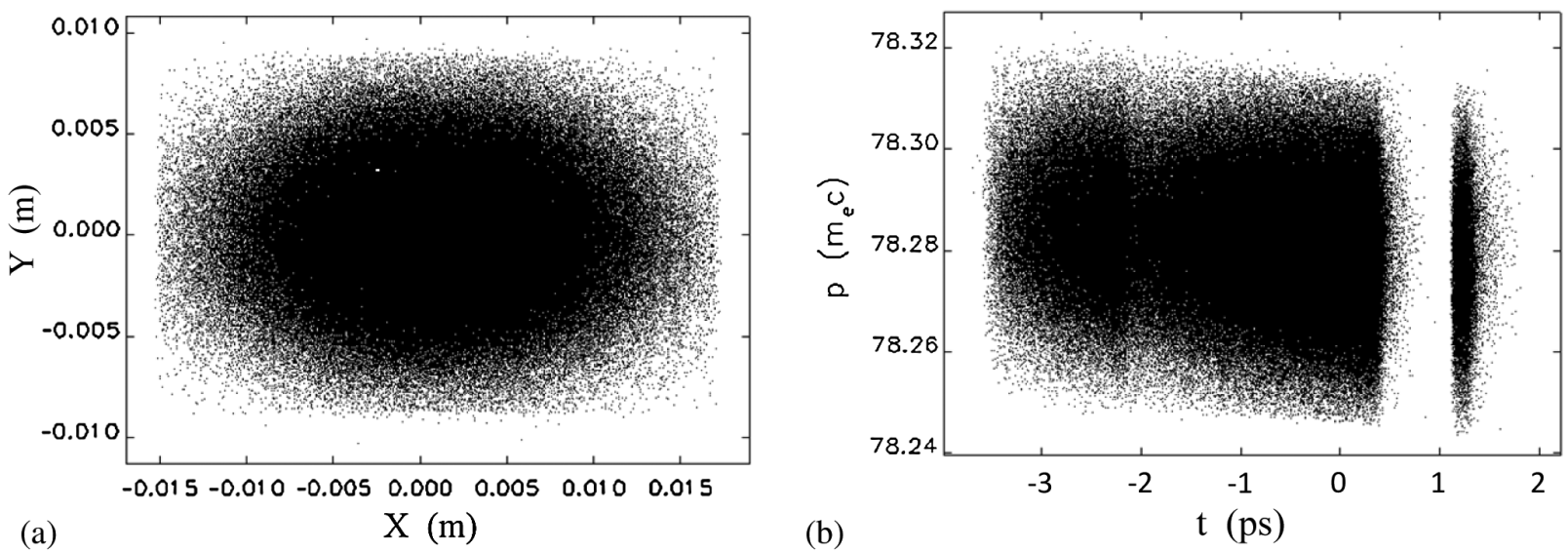

Particle flux (arb. units)

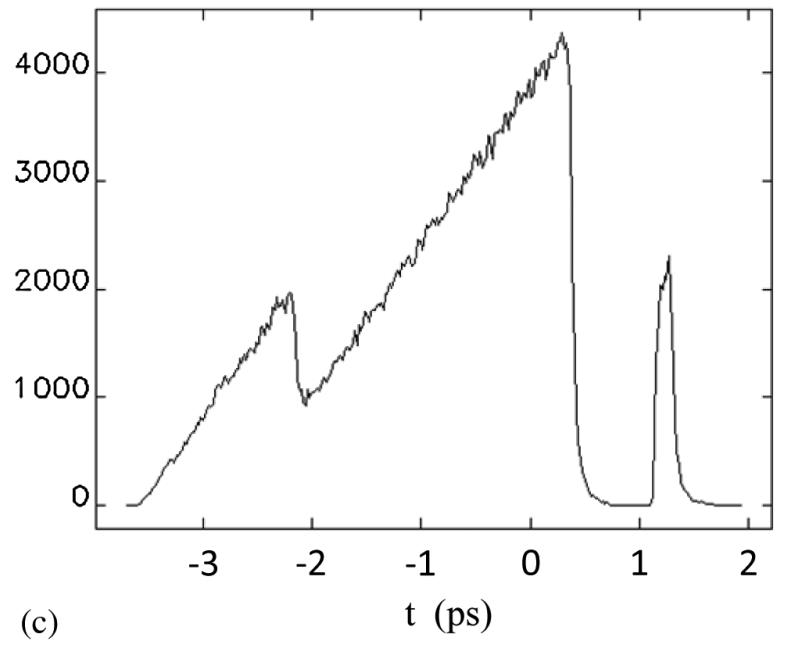

Particle flux (arb. units)

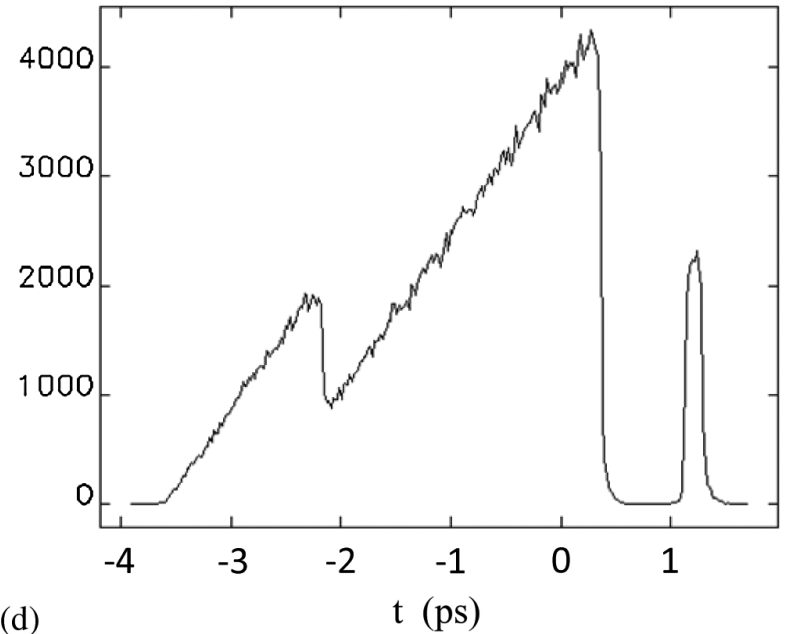

FIG. 6. Elegant third-order simulation of the EEX output beam for the input beam shown in Fig. 2: $x-y$ particles distribution (a); longitudinal momentum and arrival time particles distribution (b); beam temporal profile: without sextupole correctors (c) and with sextupole correctors (d).
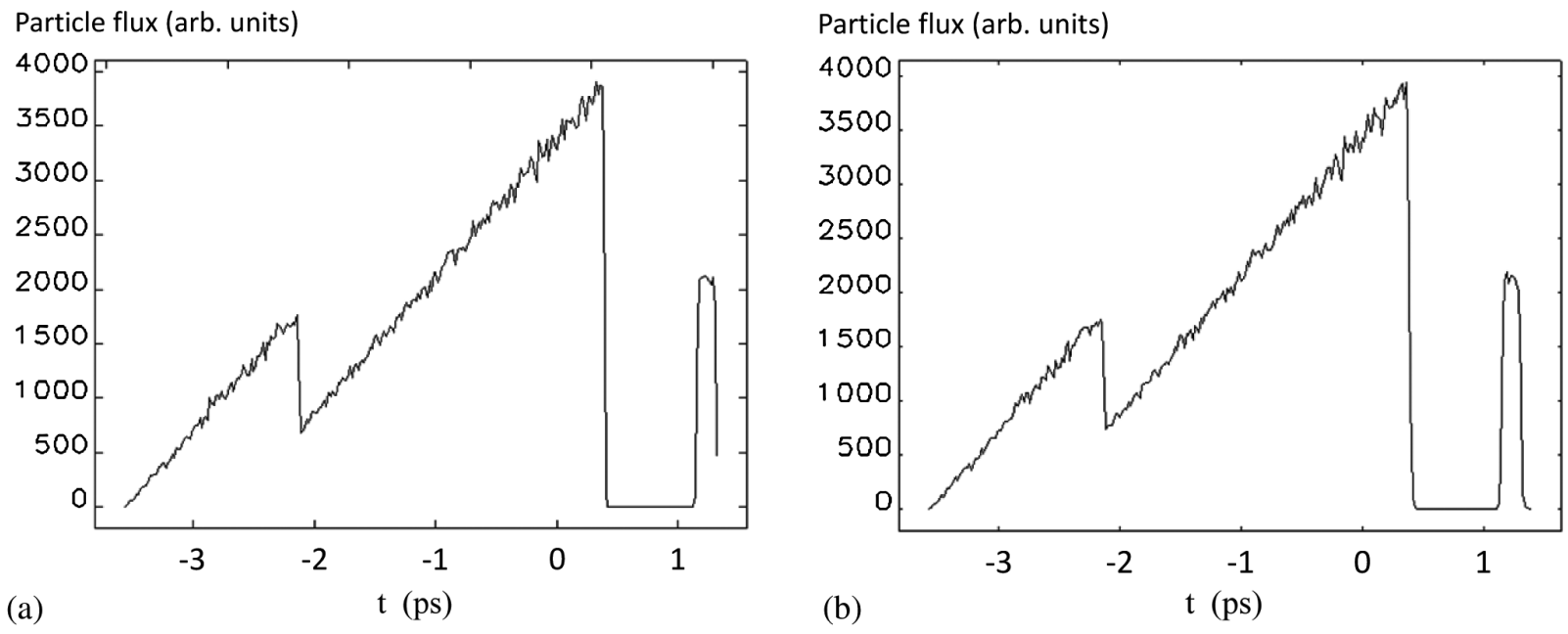

FIG. 7. Output beam in the line with sextupole correctors. Computation with a transfer matrix method accounting for the second-order (a) and up to the third-order (b) elements. 
Particle flux (arb. units)

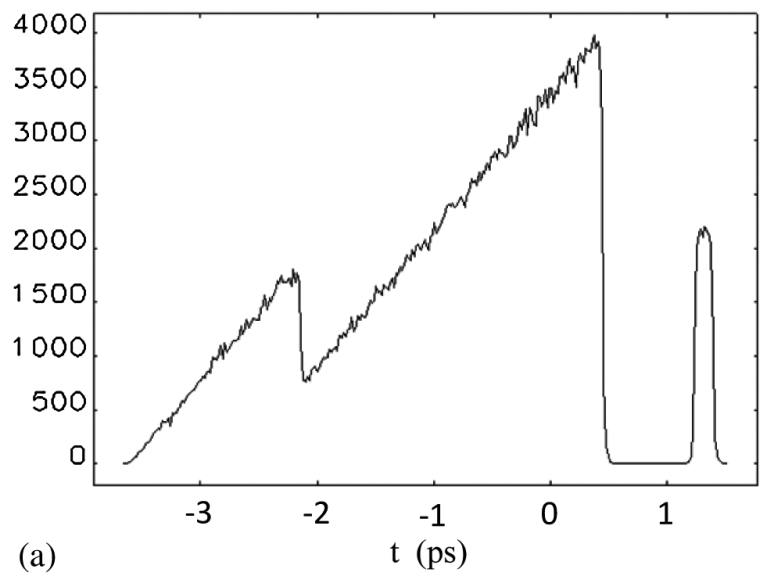

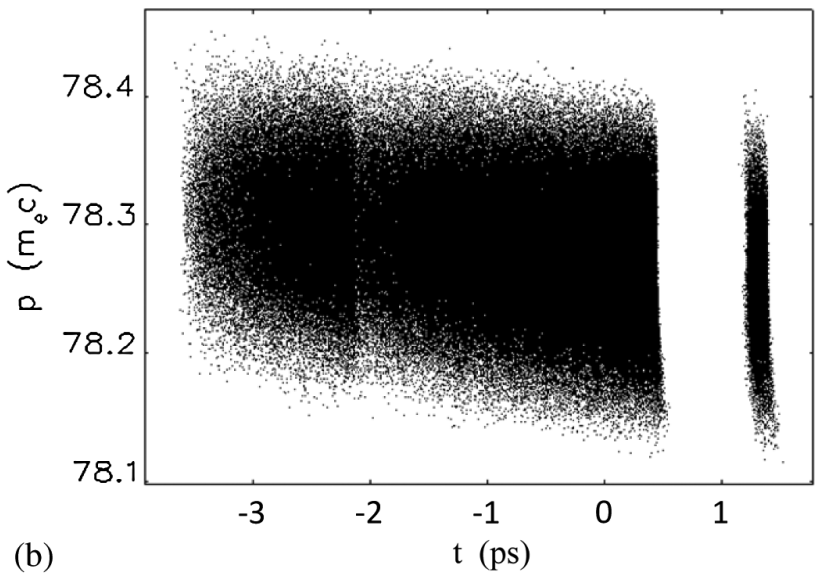

FIG. 8. Output beam, computation with a third-order real standing wave cavity matrix provided by Tech-X and imported into Elegant. Beam time profile (a) and momentum spread (b).

the second dogleg. The computed output current [Fig. 8(a)] looks very close to the case of the idealized traveling wave cavity. However, the momentum plot demonstrates an unacceptable energy spread of more than $0.3 \%$ in the witness bunch [Fig. 8(b)]. The source of it is a high absolute value of $R_{6,5}$ transfer matrix element, $R_{6,5}=-0.43 \mathrm{~m}^{-1}$, and it evidently has to do with the standing wave type of the deflecting cavity.

Another method for slight improvement of the main beam shape in particular would be shifting the beam axis and the mask as a whole along the $\mathrm{x}$ direction so that the witness bunch's trajectory goes through the beam line center. In addition, octupole magnets could be used to suppress the third-order transfer matrix elements; however, these would also make the system too complicated and difficult for aligning and tuning.

\section{CHARGE RELATED EFFECTS}

When the input beam has a charge of $0.5 \mathrm{nC}$ or greater, which corresponds to a beam current of higher than $100 \mathrm{~A}$, the charge-caused output current aberrations become noticeable and not possible to be ignored. Using the kicks method, Elegant allows for the simulation of the longitudinal space charge effect in the drift areas and the CSR effect in the dipole bends. To demonstrate how these effects change the output beam current shape we completed simulations with a $5 \mathrm{nC}$ input beam. As it can be seen from Fig. 9(a), accounting for these effects results in a distorted current profile. To separate the two effects, we simulated the longitudinal phase space of the output beam accounting for only one of these effects at a time. According to Fig. 9(b), the CSR effect induces an energy loss and a big energy spread of about $1 \%$ which in turn results in a different time lag of particles with different energies with the delay time for higher energy particles being smaller. Although in a linear order the particle's propagation time in the whole system does not depend on its energy, it is not the case for particles that lost energy inside of the beam line. It can be seen that particles that lost more energy arrive at the end of the beam line later in time and as a result it causes blurring of all details in the beam time profile. The particles in the tail of the double triangular bunch demonstrate the biggest energy loss. There is also a shorter wavelength instability observed at sharp edges. The longitudinal space charge (LSC) effect results in much smaller induced energy change increasing the initial energy spread by approximately 2 times. The LSC that caused beam current shape distortion also includes the aforementioned mechanism of the particle's propagation time dependence on the energy change. Yet, particles at sharp bunch edges contribute to the most pronounced bunch expansion.

We did not account for the transverse space charge (TSC) effect which is very different from the longitudinal space charge effect. The transverse space charge effect causes transverse beam expansion which is a generally much stronger effect than the charge caused longitudinal beam expansion. However, as opposed to the TSC, in case of an EEX the LSC has another stronger mechanism of causing aberrations through the beam energy change inside of the energy dispersive beam line. That makes a direct comparison between the transverse and longitudinal effects complicated. The TSC has to be accounted for when the assumption used in Elegant simulations that the beam transverse dynamics is emittance dominated becomes not valid. The beam dynamics is considered emittance dominated when $\varepsilon^{2} \gg K a^{2}$, where $\varepsilon$ is the beam emittance, $a$ is the beam radius and $K(I)$ is the beam perveance. For our beam with a $5 \mathrm{nC}$ charge this condition is not satisfied anywhere in the EEX beam line, thus we conclude that the TSC effect is important. However, accounting for the TSC is beyond the built-in Elegant capabilities. 

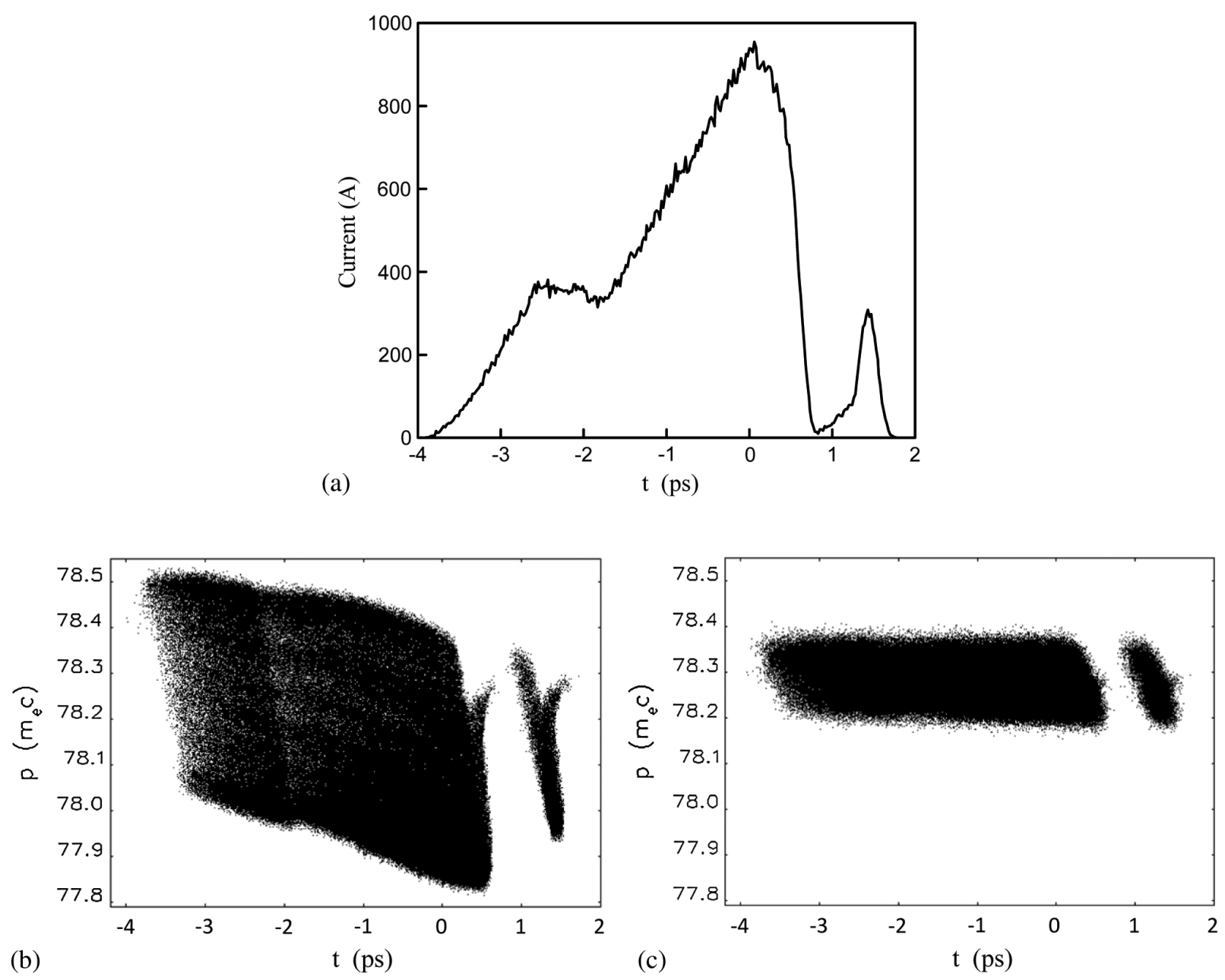

FIG. 9. Output beam current simulated in Elegant using the kicks method demonstrating aberrations caused by accounting for both the LSC effect and the CSR effect in the bends (a). Longitudinal phase space of the output beam produced by each of these effects separately: (b) for CSR, (c) for LSC. The corresponding beam charge before the mask was $5 \mathrm{nC}$ in all simulations.

For high gradient accelerators the drive bunch charge has to be high to induce a strong wakefield in the DWA: the higher the charge, the higher the accelerating gradient is. Therefore, the beam charge effects put a restriction on the gradient that can be realistically achieved without too much degradation of the accelerated bunch. We did not perform minimization of the bunch charge related effects since this would eventually interfere with the desire to have higher order transfer matrix elements small. This section, however, suggests that the CSR and space charge effects represent an issue. These effects will be studied in detail in our future experiments.

\section{CONCLUSION}

We have successfully designed and optimized two possible EEX configurations: a double dogleg and a chicane. The EEX provides a unique tool for shaping beam currents in a small time scale. We realized that this method is not perfect because the higher order nonlinear effects in the beam line distort the beam from the desired pulse shape. Suppression of nonlinearities by inserting new elements into the beam line like sextupoles or even octupoles can help reduce higher order nonlinearities, but will also increase the complexity of the system that will eventually make tuning and aligning more difficult. The other restriction of the EEX applicability is the bunch charge related effects. The coherent synchrotron radiation in dipole bends, the transverse space charge beam expansion, and the longitudinal space charge nonuniform energy change will all produce distortions in the output beam current. In contrast to the beam line nonlinearities, the charge related effects are stronger for tighter-focused beams. To fully understand the EEX performance restrictions we plan to conduct a beam shaping experiment.

\section{ACKNOWLEDGMENTS}

This work is supported by the U.S. Department of Energy through the Laboratory Directed Research and 
Development (LDRD) program at Los Alamos National Laboratory. We thank Ilya Pogorelov and Dan Abell for their help in computing the higher order matrix for LANL's deflecting cavity, John Lewellen for help with Elegant, and Quinn Marksteiner for proofreading the paper.

[1] The World Wide Web Virtual Library: Free Electron Laser research and applications, http://sbfel3.ucsb.edu/www/.

[2] S. Schreiber, B. Faatz, J. Feldhaus, K. Honkavaara, R. Treusch, and M. Vogt, FEL2012 Conference Proceedings, Nara, Japan (2012), MOPD01.

[3] J. Blau, K. Cohn, W. B. Colson, A. Laney, and J. Wilcox, FEL2012 Conference Proceedings, Nara, Japan (2012), WEPD01, [http://accelconf.web.cern.ch/AccelConf/ FEL2012/papers/wepd01.pdf].

[4] H. H. Braun, FEL2012 Conference Proceedings, Nara, Japan (2012), MOOB04.

[5] L. Giannessi, E. Allaria, L. Badano et al., FEL2012 Conference Proceedings, Nara, Japan (2012), MOOB06.

[6] MaRIE 1.0 proposal, http://marie.lanl.gov/docs/proposal .pdf.

[7] J. Rossbach, Proceedings of the 29th Free Electron Laser Conference, Novosibirsk, Russia (BINP, Novosibirsk, 2007), MOBAU02.

[8] C. Jing, A. Kanareykin, J. G. Power, M. Conde, W. Liu, S. Antipov, P. Schoessow, and W. Gai, Phys. Rev. Lett. 106, 164802 (2011).

[9] MaRIE website, http://marie.lanl.gov/.

[10] R.W. Garnett, M.S. Gulley, Proceedings of the 25th International Linear Accelerator Conference, LINAC-2010, Tsukuba, Japan (KEK, Tsukuba, Japan, 2010), TUP038.

[11] B.E. Carlsten, C. W. Barnes, K. A. Bishofberger et al., Proceedings of the 24th Particle Accelerator Conference, PAC-2011, New York, 2011 (IEEE, New York, 2011), TUODS1.

[12] W. Gai, P. Schoessow, B. Coley, R. Konecy, J. Norem, J. Rosenzweig, and J. Simpson, Phys. Rev. Lett. 61, 2756 (1988).

[13] M. Rosing and W. Gai, Phys. Rev. D 42, 1829 (1990).

[14] M. C. Thompson, H. Badakov, A. M. Cook, J. B. Rosenzweig et al., Phys. Rev. Lett. 100, 214801 (2008).

[15] C. Jing, W. Gai, J. G. Power, R. Konecny, W. Liu, S. H. Gold, A. K. Kinkead, S. G. Tantawi, V. Dolgashev, and A. Kanareykin, IEEE Trans. Plasma Sci. 38, 1354 (2010).

[16] M. E. Conde, Proceedings of the 22nd Particle Accelerator Conference, PAC-2007, Albuquerque, NM (IEEE, New York, 2007), pp. 1899-1903.
[17] E. I. Simakov, B. E. Carlsten, D. Shchegolkov, AIP Conf. Proc. 1507, 634 (2012).

[18] K. L. F. Bane, P. Chen, and P. B. Wilson, IEEE Trans. Nucl. Sci. 32, 3524 (1985).

[19] P. B. Wilson, SLAC Report No. 296, 1985, pp. 273-295.

[20] G. V. Sotnikov and T. C. Marshall, Phys. Rev. ST Accel. Beams 14, 031302 (2011).

[21] C. Jing, A. Kanareykin, J. G. Power, M. Conde, Z. Yusof, P. Schoessow, and W. Gai, Phys. Rev. Lett. 98, 144801 (2007).

[22] B. Jiang, C. Jing, P. Schoessow, J. Power, and W. Gai, Phys. Rev. ST Accel. Beams 15, 011301 (2012).

[23] The workshop on application of dielectric wakefield accelerators to next generation $\mathrm{x}$-ray free-electron laser facilities, Argonne, IL, https://twindico.hep.anl.gov/indico/ conferenceTimeTable.py?confId=428.

[24] M. Cornacchia, S. Di Mitri, G. Penco, and A. A. Zholents, Phys. Rev. ST Accel. Beams 9, 120701 (2006).

[25] P. Muggli, V. Yakimenko, M. Babzien, E. Kallos, and K. P. Kusche, Phys. Rev. Lett. 101, 054801 (2008).

[26] S. Antipov, C. Jing, A. Kanareykin, J. E. Butler, V. Yakimenko, M. Fedurin, K. Kusche, and W. Gai, Appl. Phys. Lett. 100, 132910 (2012).

[27] Y.-E. Sun, P. Piot, A. Johnson, A. H. Lumpkin, T. J. Maxwell, J. Ruan, and R. Thurman-Keup, Phys. Rev. Lett., 105, 234801 (2010).

[28] P. Emma, Z. Huang, K.-J. Kim, and P. Piot, Phys. Rev. ST Accel. Beams 9, 100702 (2006).

[29] M. Cornacchia and P. Emma, Phys. Rev. ST Accel. Beams 5, 084001 (2002).

[30] D. Xiang and A. Chao, Proceedings of the 24th Particle Accelerator Conference, PAC-2011, New York, 2011 (IEEE, New York, 2011), WEP044.

[31] R. P. Fliller III, H. Edwards, J Ruan, T. Koeth, Proceedings of the 11th European Particle Accelerator Conference, Genoa, 2008 (EPS-AG, Genoa, Italy, 2008), THPC013.

[32] J. Ruan, A. S. Johnson, A. H. Lumpkin, R. Thurman-Keup, H. Edwards, R. P. Fliller, T. Koeth, and Y.-E Sun, Phys. Rev. Lett. 106, 244801 (2011).

[33] C. R. Prokop, P. Piot, B. E. Carlsten, M. Church, Nucl. Instrum. Methods Phys. Res., Sect. A 719, 17 (2013).

[34] Elegant code distribution web page, http://www.aps.anl .gov/Accelerator_Systems_Division/ Accelerator_Operations_Physics/software.shtml\#elegant.

[35] M. Borland, Presented at the 6th International Computational Accelerator Physics Conference, ICAP2000, Darmstadt, Germany, 2000.

[36] Tech-X company web page, https://www.txcorp.com/. 